\title{
LA NARRATIVA ITALIANA IN \\ RUSSIA TRA LA FINE DEL XX \\ SECOLO E L'INIZIO DEL XXI
}

giulia.baselica@unito.it

Università degli

Studi di Torino

\begin{abstract}
Il contributo intende proporre una panoramica delle opere letterarie italiane contemporanee tradotte in lingua russa, nel tentativo di individuare le tendenze e gli orientamenti, quindi i generi apprezzati dal pubblico e dalle élites culturali. Il focus temporale si colloca nel periodo compreso fra gli ultimi decenni del XX secolo e i primi due decenni del nuovo Millennio, quindi in un contesto di profondi cambiamenti non solo ideologici e politici, bensì anche sociali e culturali. La produzione letteraria italiana contemporanea si innesta nell' humus culturale e letterario russo, caratterizzato dall'espressione di nuovi e peculiari generi non ammessi ufficialmente nell'epoca sovietica.

Scopo della breve indagine sarà l'identificazione di una sorta di mappa della ricezione del testo culturale italiano contemporaneo nel suo insieme, a sua volta determinata, nell'essenza, dalle tendenze letterarie prevalenti nel contesto culturale russo, ove si caratterizza il profilo e la missione culturale delle principali case editrici russe interessate alla diffusione della letteratura italiana in Russia.
\end{abstract}

Parole chiave: letteratura italiana, traduzione, editoria russa, letteratura russa

La notte del 31 dicembre 1991 si chiude un lungo capitolo della storia della Russia: l'Urss cessa formalmente di esistere. E l'iniziale illusione di libertà di consumo resa possibile dal nuovo capitalismo trova immediato riflesso in un repentino mutamento dei costumi culturali generando un clima di diffusa euforia, dominato dalla convinzione che «tutto [debba] e [possa] rinascere e trasformarsi» (Caramitti 2010: 178). L'axis mundi delle lettere russe subisce una rapida, quanto inattesa oscillazione, dando luogo, nella nuova società russa, a una profonda trasformazione dell'assetto culturale. La letteratura perde, forse per sempre, il ruolo di corifeo della cultura; scompare la figura dello scrittore professionista, maestro di pensiero ed emanazione del potere, per lasciare il posto a un'autorità dalla natura composita e influentissima, la televisione, capace di esercitare un dominio tanto incontrastabile quanto, talvolta, impercepito. Nella nuova società russa, caratterizzata da una logica consumistica, il libro, oggetto culturale, diviene anche, nell'immaginario collettivo, un bene che produce redditività.

Appaiono quindi nuovi editori che propongono generi ufficialmente ignoti ai lettori sovietici solo pochi anni prima: accanto al genere fantasy, 
al romanzo rosa, al giallo, oltre al genere erotico, al trash e, all'estremo opposto, alla produzione di carattere mistico, si affermano altri generi, che già avevano cominciato a prendere forma nella seconda metà degli anni Ottanta. Tra questi l'antiutopia, spesso dalla fisionomia grottesca, edificata sulle fondamenta della storia nazionale e della contemporaneità e più volte ispiratrice di letture controfattuali del passato - ne è un esempio il romanzo Rommat, di Vjačeslav P'ecuch, pubblicato nel 1990 - o il genere politico-letterario, non di rado voce delle tendenze antigovernative degli anni gorbačëviani, rappresentato in particolare dai romanzi di Aleksandr Zinov'ev : Moj dom-moj čužbina (La mia casa è il mio esilio, 1991); Katastrojka (2003); Iskušenie (Tentazione, 1991), e anche dal romanzo di Vasilij Aksënov, Ostrov Krym (L'isola di Crimea) - pubblicato in Urss nel 1990 e apparso già nove anni prima negli Stati Uniti - o il genere memorialistico - di cui sono espressione i Dnevniki (Diari) di Vladimir Gusev, pubblicati tra il 1995 e il 1999 e il romanzo Otstuplenie ot romana. V sezon zasolki ogurcov (Digressione dal romanzo. Nella stagione della salatura dei cetrioli), edito nel 1994, di Sergej Esin, nel quale i fatti biografici dell'io narrante vengono trasformati in fatti d'arte, oggetto di analisi e di riflessioni condotte dall'io narrante stesso - e il genere filosofico, rappresentato, per esempio, dall'opera Zateej. Iz tetradi o Nikolae Rubcove (Comincia. Dal quaderno di Nikolaj Rubcov), di Viktor Astaf'ev, pubblicata nel 2002 (Mineralov 2002).

La letteratura post-sovietica rappresenta, nell'essenza, un fenomeno storico-letterario atto a trasformare la letteratura sovietica e la letteratura della perestrojka, portando a definitivo compimento i percorsi della letteratura russa del XX secolo e, nel contempo, determinando l'inizio della produzione letteraria del XXI. Questa costituisce un fenomeno non cronologico, bensì ideologico, in quanto comprende proprio le opere che, per i principi strutturali ed espressivi da cui sono caratterizzati, si distinguono dalla produzione sovietica e diffondono le voci di quegli scrittori che non hanno accolto i dogmi del realismo socialista, e che già in epoca sovietica erano riusciti a pubblicare, in parte utilizzando i canali ufficiali, in parte avvalendosi dell'editoria clandestina (samizdat e tamizdat). La letteratura post-sovietica vive dunque una vita parallela a quella sovietica già negli anni Sessanta, nei circoli e nei gruppi non ufficiali, nelle letture private, nelle esibizioni organizzate negli appartamenti, e nell'ultimo decennio del XX secolo essa trova espressione anche nei generi tradizionali, incarnati da prosatori e poeti di varie generazioni, come Aleksandr Solženicyn, Ljudmila Petruševskaja, Vladimir Makanin, Oleg Čuchoncev, Maksim Amelin; in molteplici orientamenti, come il postmodernismo e il concettualismo, il realismo socialista e il neobarocco, che connotano

1Varie le possibili specificità e varianti di tale genere: il romanzo di costume, del quale Aleksandra Marinina è uno degli autori più rappresentativi; il romanzo pseudo-storico, oggetto di interesse per B.Akunin; il romanzo politico per le opere di Lev Gurskij ed Eduard Topol'; il thriller, genere praticato, per esempio, da Viktor Docenko (Ivanova 2007). 
la prosa di Andrej Bitov, Saša Sokolov, Tat'jana Tolstaja e la prosa saggistica di Dmitrij Galkovskij; nella letteratura d'intrattenimento, la cui voce più rappresentativa è forse quella di Ljudmila Ulickaja; nel neonaturalismo, come evoluzione della tradizione del realismo - l'umorismo nero della prosa di Sergej Kaledin, la nuova prosa femminile di Svetlana Vasilenko, Inna Gorlanova, Marina Palej - unito al neosentimentalismo di Aleksej Slapovskij e di Marina Vyšneveckaja; nel postrealismo come nuova estetica artistico-letteraria - ne è un esempio l'autobiografismo della produzione teatrale e narrativa di Evgenij Griškovec - o nel nuovo realismo dei giovani scrittori, come Oleg Pavlov, Roman Senčin, Sergej Šargunov; nell'ipernaturalismo della nuova drammaturgia russa, rappresentata, per esempio, dalle opere di Michail Ugarov (Ivanova 2007).

Nel corso degli anni Novanta la produzione postmodernista assurge alla posizione e al ruolo di genere letterario di moda, mentre alla produzione che si pone in linea di continuità con la tradizione si sostituisce da una parte l'affermazione della cultura di massa, dall'altra la letteratura postmoderna, il cui tratto identitario essenziale coincide, innanzitutto, con un evidente processo di deideologizzazione: la discussione ideologica e politica, per la quale la letteratura dell'epoca sovietica era tema e occasione, si trasforma ora in discussione letteraria (Ivanova 2007).

La letteratura postsovietica è interessata da un processo di erosione del ruolo della voce narrante onnisciente, dalla dispersione e contrazione dei tradizionali generi prosastici e poetici (romanzo, povest', racconto, poema, lirica), sui quali si affermano forme e generi ibridi. Tratto distintivo dell'intertestualità del postmodernismo russo è il principio del rizoma, generatore di generi, di note alle note, di cataloghi interamente costituiti di opere essenzialmente formate da infiniti commenti: ne sono esempi i romanzi Bezkonečnyj tupik (Il vicolo cieco infinito), di Dmitrij Galkovskij, Blizkoe retro (Il retro vicino) di Andrej Bitov, Podlinnaja istorija zelënych muzykantov (La vera storia dei musicisti inesperti) di Evgenij Popov; di serie di involucri che racchiudono svariate narrazioni come Linii susd'by, ili sunduk Milašsvič di Mark Charitonov, Albom dlja marok (Album di francobolli) di Andrej Sergeev, di raccolte di commenti assimilabili, per volume e per estensione, a vere e proprie narrazioni, come Rasstavanie s Narcissom (Separazione da Narciso) di Aleksandr Gol'dštejn. Al confine tra fiction e non-fiction, tra letteratura documentale e saggistica, compaiono testi nei quali vengono sperimentate le più svariate possibilità stilistiche, come Konec citaty (Fine della citazione) di Michail Bezdomnyj, o i più diversi generi ibridi, come la prosa filologica di Aleksandr Genis (Ivanova 2007).

In tale contesto, complesso e a tratti variegato da tendenze indefinite e, non di rado, contraddittorie, in questo nuovo momento - evocando la lezione lotmaniana - di esplosione, di spinta propulsiva, atta ad assicurare, appunto, l'innovazione, al quale si integra il graduale processo della continuità e della tradizione, può essere interessante tentare di individuare la presenza della letteratura italiana, in particolare della produzione 
narrativa contemporanea, ricordando che «la dinamicità della cultura è frutto della coesistenza, all'interno di un medesimo spazio culturale, di diverse lingue, legate da gradi diversi di affinità e traducibilità, 0 , viceversa, di estraneità e intraducibilità» (Lotman 1994: 34).

Così, nello spazio culturale postsovietico dei primi anni Novanta si osserva, innanzi tutto, la presenza di un'evidente linea di continuità con il dialogo letterario fra la cultura russa e la cultura italiana. Negli anni immediatamente successivi al raspad, alla dissoluzione dell'Urss, cioè nel triennio 1992-'94, si continua infatti a pubblicare le opere di autori ampiamente noti al pubblico russo, come Luigi Pirandello e Alberto Moravia. Del primo viene pubblicata, nel ' 94 , dalla casa editrice moscovita Panorama, una raccolta di opere scelte (Izbrannye proisvedenija, trad. I. Volodina) che comprendono Il fu Mattia Pascal e alcune novelle e opere teatrali ${ }^{2}$. Di Moravia compaiono nel '92 una nuova edizione de Gli indifferenti (Ravnodušnye, trad. L. Veršinin, Rjazan, Russkoe slovo), dei romanzi La Romana e Il disprezzo ( Rumljanka, Prezrenie, trad. I.Gusev, Moskva, Press), La noia (Skuka, trad. S. Bušueva, Sankt Peterburg, Chudožestvennaja literatura. Nel '93 le edizioni Veče di Mosca pubblicano una nuova versione de Gli indifferenti e de La Romana (trad. I.Zaslavskaja) e l'anno seguente la casa editrice Press edita una raccolta che comprende il romanzo Il conformista e due racconti, Agostino e La provinciale (Konformist. Roman i dve povesti, a cura di R. Rybkin) mentre Respublika di Mosca propone nella traduzione di G.Kiselev, il romanzo Io e lui (Ja i on) ${ }^{3}$. Interessante è il caso di Sciascia: nel ' 92 viene pubblicato un ponderoso volume, una raccolta intitolata Morte dell' inquisitore (Smert' inkvizitora, Moskva, Progress) e costituita da numerosi romanzi brevi (La strega e il capitano; Il Consiglio d'Egitto; La scomparsa di Majorana; La morte di Stalin; Todo modo; Il cavaliere e la morte; Una storia semplice), alla quale nei decenni successivi non seguiranno nuove edizioni di opere dello scrittore siciliano, tuttavia piuttosto noto in epoca sovietica. Nel '67 l'edi-

2Seguiranno, nel 2006, per i tipi della EKSMO di Mosca la raccolta I tre pensieri della sbiobbina (Tri mysli gorbun'i: roman, novelly i p'esy) che contiene Il fu Mattia Pascal, alcune novelle e i drammi Sei personaggi in cerca d' autore ed Enrico IV, e nel 2011, nelle edizioni Tekst, la versione russa dei Quaderni di Serafino Gubbio operatore (Zapiski kinooperatora Serafino Gubbio, trad. V. Luk'jančuk). L'opera pirandelliana era apparsa nel contesto culturale sovietico nel 1958 con una raccolta di novelle (Novelly, trad. N.Medvedev, Moskva, Golitizdat) e nel '67 con Il fu Mattia Pascal (Pokojnyj Mattia Pascal, trad. G. Rubcova e N. Rikova, Leningrad, Chudožestvennaja literatura), otto anni dopo la stessa casa editrice e le stesse traduttrici approntarono la versione russa de I vecchi e i giovani (Starye i molodye); nell'83 uscì una raccolta di prose scelte in due volumi (Izbrannaja proza $v 2 \mathrm{ch}$ tomach, Leningrad, Chudožestvennaja literatura) e, nell'86, fu la volta di una nuova raccolta di novelle (Novelly) presso la casa editrice Chudožestvennaja literatura di Mosca. (Pavan 2014)..

3Le opere di Moravia, che seguiteranno a essere pubblicate anche nel primo decennio del XXI secolo, erano in parte note al lettore sovietico già a partire dagli anni Cinquanta del secolo scorso. All'epoca dell'ottepel', del disgelo poststaliniano, risalgono infatti i Racconti romani, preceduti da una prefazione firmata dallo scrittore Il'ja Erenburg (Rimskie rasskazy, Moskva, Izdanie inostrannoj literatury, 1956) e altri racconti comparvero in varie edizioni nel corso degli anni Sessanta e Settanta. 
trice Pravda di Mosca aveva dato alle stampe il racconto La zia d'America (Amerikanskaja tetuška) e nell'82 l'edizione simultanea di due raccolte avevano permesso al pubblico russo di conoscere piuttosto approfonditamente la produzione di Leonardo Sciascia: Il mare colore del vino (Vinnogo cveta more: romany, povesti, rasskazy, Moskva, Progress) con le opere Il consiglio d'Egitto; A ciascuno il suo; I pugnalatori, L'antimonio, Il mare colore del vino; Filologia; Western di cose nostre e la raccolta I pugnalatori (Palermskie ubijcy: povesti, Moskva, Izvestija), con, oltre al racconto omonimo, Il mare colore del vino 4 . Si tratta di un autore, fra i classici italiani del Novecento, evidentemente apprezzato nell'epoca che aveva preceduto la dissoluzione dell'Urss forse soprattutto per le tematiche affrontate nei suoi racconti e romanzi $i^{5}$ e per il suo credo politico, e in seguito dimenticato.

Altro importante classico italiano del Novecento, Dino Buzzati, si afferma nella dimensione culturale e letteraria russo-sovietica negli anni della perestrojka, quando il tono fiabesco e surreale della sua visione del mondo, la trasfigurazione del quotidiano, la tendenza all'allegorismo trovano spazio fra i vincoli, ormai allentati, del realismo socialista; nel 1985 le edizioni Izvestija pubblicano la raccolta I sette messaggeri (Sem' goncov); quattro anni dopo la casa editrice Raduga edita in un unico volume Il deserto dei tartari; Il grande ritratto e una scelta di racconti (Tatarskaja pustyn' ja; Uveličennyj portret; Rasskazy) e, nel '91, appare per la moscovita Kul'turnaja iniciativa la raccolta Sessanta racconti (Linkor smerti). Dal '93, con Un amore (Ljubov', trad. U. Ilyn, Sankt Peterburg, Peterburg XXI) al 2011, con una nuova edizione dei Sessanta racconti (Sest'desjat rasskazov, Moskva, Astrel') i principali scritti buzzatiani vengono periodicamente ripubblicati ${ }^{6}$.

Se il decennio successivo al raspad può essere considerato, in merito al clima culturale, quindi anche alla ricezione della letteratura italiana, una sorta di prolungamento dell'età breve della perestroj $k \mathrm{a}^{7}$ - con il nuovo interesse per le manifestazioni della spiritualità e dell'inconscio, con un conciliante atteggiamento nei confronti della religione, infine con, in un ambito affatto diverso, la liberalizzazione dei generi popolari e degli argomenti un tempo tabù, come la criminalità, la droga e la prostituzione (Kozack 1994) - la presenza di particolari voci italiane nel polisistema letterario russo

4Vi era poi la circolazione in samizdat di alcune opere non ammesse alla diffusione ufficiale, come il romanzo La scomparsa di Majorana, accusato di contenere allusioni al caso Sacharov. (Dobrovolskaja 2006).

5La voce Sciascia Leonardo in Sovremennaja Enciklopedija rileva il carattere di giallo politico delle sue narrazioni, dedicate alla vita in Sicilia, orientate a tracciare un quadro delle malattie del sistema, quali la corruzione dell'apparato statale, i crimini compiuti dalla mafia, ecc.

6Una rapida navigazione in rete fra i forum e i blogs (come I book you, Schizonet, Klub čitaem vmeste) permette di constatare il sentimento di meraviglia suscitata nei lettori odierni dalla lettura del romanzo Il deserto dei tartari.

7Una sintesi delle tendenze letterarie liberate nell'età della perestrojka può essere riconosciuta in tre romanzi: Pečal'nyj detektiv (Un triste romanzo giallo) di Viktor Astaf'ev; Placha (Il patibolo) di Čingiz Ajtmatov; Moskovskaja krasavica (La bella di Mosca) di Viktor Erofeev, tuttavia concepiti e scritti prima del 1985. 
conferma il radicamento di nuove istanze che si manterranno nel periodo successivo. Compare l'edizione, in volume singolo, del romanzo La donna della domenica di Fruttero e Lucentini (Ego osenilo v voskresen'e, trad. L. Veršinin, Chabarovsk, RIOTIP, 1992); escono alcuni fra i più noti romanzi di Giorgio Scerbanenco (Venere privata; Traditori di tutti; I ragazzi del massacro; I milanesi ammazzano il sabato), raccolti in un volume dal titolo Milancy ubivajut po subbotam (trad. I.Zaslavskaja, Moskva, Voschoždenie, 1992), poi riedito con un altro titolo (Junye sadisty, Moskva, Centrpoligraf, 1994); vengono pubblicate le raccolte Il poliziesco italiano contemporaneo ${ }^{8}$ (Sovremennyj ital'janskij detektiv, Moskva, Raduga, 1993) con i romanzi Giro di voci di Vieri Razzini e Il sospetto di Laura Grimaldi; e I giovedi della signora Giulia (Soblazny Džulii: Ital' janskij detektiv: Romany, Moskva, InterDigest, 1993) con i romanzi I giovedi della signora Giulia di Piero Chiara; Picnic con sangue di Renato Olivieri; Morte per lucro di Carlo Villa. In questi anni, nel genere sentimentale, debutta la produzione di Sveva Casati Modignani: nel '95 appaiono ben tre romanzi tutti pubblicati dalla Eksmo di Mosca: Come stelle cadenti; E infine una pioggia di diamanti e Donna d'onore (Kak padajuščie zvezdy, trad. I. Čelyševa; Naslednica brilliantov, trad. E. Dmitrieva; Nevesta nasilija, trad. E. Moločkovskaja e I. Čelyševa. La narrativa poliziesca italiana nel genere sentimentale, pur mediante voci e fisionomie diverse, continuerà a proporsi ai lettori russi fino alla fine del secondo decennio del nuovo secolo. A Fruttero e Lucentini e Scerbanenco si sostituiranno Giorgio Faletti - con i romanzi Io uccido (Ja ubivaju, trad. I. Konstantinova, 2005 e 2007); Niente di vero tranne gli occhi (Narisovannaja smert': Glaza ne lgut nikogda, trad. I. Zaslavskaja, 2006 e 2008) editi dalla pietroburghese Azbuka-klassika e Fuori da un evidente destino (Ubijstvennaja ten', trad. I. Konstantinova, 2008), pubblicato da Astrel' di Mosca - e Andrea Camilleri, i cui romanzi La forma dell'acqua; Il cane di terracotta; Il ladro di merendine; La concessione del telefono; La voce del violino (Forma vody, trad. A. Kondjurina; Sobaka iz terrakotty, trad. A. Kondjurina; Pochititel' škol'nych zavtrakov, trad. K. Denisovič; Telefon, trad. E. Solonovič; Golos skripki, trad. E. Čemogina) verranno immessi nel mercato editoriale russo dalla moscovita Inostranka, tra il 2004 e il 2007, mentre la casa editrice Mir knigi, anch'essa di Mosca, pubblicherà nel 2007 La pensione Eva (Pansion Evy, trad. E. Kazmin). A tale genere danno voce italiana anche opere di altri autori'. I romanzi sentimentali, che trovano nelle vicende

8 Nel 1980 era già comparsa, per i tipi della casa editrice Progress, una raccolta con lo stesso titolo e contenente i romanzi La donna della domenica e La morte con le ali bianche di Felisatti-Pittorru.

9Fra queste ricordiamo Il passato è una terra straniera di Gianrico Carofiglio (Prošloe-čužaja zemlja, trad. E. Kislova, Moskva, Inostranka); La collega tatuata (Smertel'nyj urok, trad. Ju. Šujska, Moskva, Mir knigi, 2008); Lupo mannaro, Almost blue, Un giorno dopo l'altro di Carlo Lucarelli (Obototen', Almost blue, Den' za dnem, trad. A. Miroljubova, Sankt Peterburg, Azbuka-klassika). Di recente acquisizione da parte del mercato editoriale è la produzione di Donato Carrisi, con La ragazza nella nebbia (Devuška v tumane, Azbuka, 2017, trad. di O.Egorov); Il tribunale delle anime (Poterjannye devuški Rima, Azbuka, 2017, trad. di A.Miroljubova; Il cacciatore del buio (Ochotnik za ten' ju, Azbuka, 2018, trad. di A. Miroljubova); 
narrate da Sveva Casati Modignani l'espressione italiana più popolare - dal 1995 al 2004 numerosi titoli, tra quelli prodotti dalla coppia di scrittori celata dietro tale pseudonimo, vengono pubblicati con tirature non inferiori alle cinquemila copie, per lo più dalla casa editrice Eksmo di Mosca - sono rappresentati da svariati autori che godono vasta popolarità in patria ${ }^{10}$.

Ma il segno anticipatore di un'autentica svolta nella ricezione della letteratura italiana da parte del contesto russo si esprime nella pubblicazione, già nel 1989, del romanzo Il nome della rosa di Umberto Eco ${ }^{11}$ (Imja rozy, trad. E. Kostjukovič, Moskva, Kniga Palata, con postfazione di Jurij Lotman) ${ }^{12}$, romanzo considerato tipicamente postmoderno - quale «traduzione, grazie ad un programmatico double coding tipicamente postmoderno, dell'esperienza neoavanguardistica in termini di una perfetta comunicabilità» (Palumbo 2011) - con la sua marcata connotazione intertestuale, l'ironica rivisitazione del passato, l'inclusione, nell'impianto strutturale stesso, di riferimenti alle coeve ricerche semiotiche. Il notissimo romanzo di Umberto Eco parrebbe offrirsi alle lettere russe come una sorta di collocutore di un altro romanzo postmoderno, russo, Puškinskij dom (La casa di Puškin) di Andrej Bitov, pubblicato nel 1978 dalle statunitensi edizioni Ardis, di Ann Arbor - e Il nome della rosa esce in Italia nel 1980 - ed edito in Russia nell'89. Al dialogo postmoderno fra Eco e Bitov si unisce, nell'anno 2000, Calvino $^{13}$ con Se una notte d'inverno un viaggiatore (Esli odnaždy zimnej noč ju putnik, trad. G. Kiselev, Moskva, Inostrannaja literatura) ${ }^{14}$.

\section{Il suggeritore (Podskazčik, Azbuka, 2018, trad. di I. Zaslavskaja).}

10Figurano, per esempio, Federico Moccia, con Tre metri sopra il cielo (Tri metra nad nebom, trad. A. Gerasimov, Sankt Peterburg, Moskva, Limbus-press, 2006; 2012; 2013); Melissa Panarello, con L'odore del tuo respiro (Aromat tvoego dychanija, trad. Ju. Šujska, Moskva, Mir knigi, 2008); Fabio Volo, con i romanzi Il giorno in più; Un posto nel mondo; È una vita che ti aspetto, tutti pubblicati dalla casa editrice RIPOL di Mosca (Improvizacija na temu ljubvi, trad. N. Bogdanov, 2009, ripubblicato nella stessa versione e dallo stesso editore ma con il titolo Ešče odin den', nel 2011; Moe bol'šoe malen'koe Ja, trad. N. Bogdanov; Vsju žizn' ja ždu tebja, trad. N. Sergeeva, 2011).

$11 E c o$ è l'autore italiano che conta il maggior numero di opere pubblicate in Russia, oltre al già citato romanzo Il nome della rosa ricordiamo: A passo di gambero; Baudolino; Cinque scritti morali; Come si fa una tesi di laurea; Il cimitero di Praga, Il pendolo di Foucault; In che cosa crede chi non crede? [con C.M. Martini]; La bustina di Minerva; L'isola del giorno prima; La misteriosa fiamma della regina Loana; La ricerca della lingua perfetta nella cultura europea; Le poetiche di Joyce; Non sperate di liberarvi dei libri [con J.C. Carrière]; Sei passeggiate nei boschi narrativi; Storia della bruttezza; Storie delle terre e dei luoghi leggendari; Vertigine della lista. Per le indicazioni bibliografiche relative ai titoli citati si rimanda il lettore al volume L'Italia in cirillico, cit. Nel 2018 appare inoltre Come viaggiare con un salmone (Kak putešestvovat's lososem, Slovo, trad. di N. Kuliš).

12Imja rozy verrà pubblicato, con continue ristampe, dal 1999 al 2010 dalla casa editrice Symposium di San Pietroburgo e nel 2011 dalla moscovita Astrel'.

13 Di Calvino erano già apparsi numerosi titoli, a partire dal 1965 con Il barone rampante (Baron na dereve, trad. L. Veršinin, Moskva, Chudožestvennaja literatura). Nel 2000 viene data alle stampe una raccolta di opere scelte in tre tomi per i tipi della pietroburghese Symposium. $14 \mathrm{~A}$ questi due autori si riconduce, nell'essenza, la produzione postmoderna italiana. Os- 
L’anno 2001 inaugura una fase nuova e interessante del dialogo fra le due letterature: l'editoria russa accoglie le opere di tre autori fra loro piuttosto diversi, ognuno espressione di un orientamento distinto. Compare la prima traduzione di Se questo è un uomo di Primo Levi ${ }^{15}$ (Čelovek li eto?, trad. E. Dmitrieva, Moskva, Družba narodov), opera che si inserisce nel contesto russo della produzione letteraria ispirata dall'universo concentrazionario e costituita da opere eterogenee ${ }^{16}$, ma prevalentemente di carattere memorialistico - come Krutoj maršrut di Evgenija Ginzburg (Viaggio nella vertigine, in «Daugana», nn. 7-12, 1988; nn. 1-6, 1989, in volume, Moskva, 1990); Nepridumannoe di Lev Razgon (La nuda verità, in «Ogonëk», 44, 1988; in volume, Moskva, 1988 ); Zemlja rodnaja di Dmitrij Lichačëv (La mia Russia, Moskva, 1983) - il cui modello archetipico può essere identificato, innanzitutto, in uno scritto nel quale la memoria personale si ibrida con la fiction, il romanzo breve Una giornata di Ivan Denisovič di Aleksandr Solženicyn, pubblicato nel 1962.

Con la traduzione, approntata per entrambi i romanzi dallo stesso traduttore, G.Kiselev editi da Inostranka di Mosca, di Oceano mare (More-okean) e di Seta (Šëlk), autentico successo editoriale, perché ripubblicato quasi ogni anno, fino al 2018, Alessandro Baricco debutta sulla scena letteraria russa, apportando la fisionomia composita della propria cospicua ed eclettica produzione, ascrivibile a più generi: non-fiction, autofiction, narrativa di ispirazione postmodernista, saggistica ${ }^{17}$. Il terzo scrittore italiano atto a rappresentare nel panorama culturale russo, nell'anno 2001, un'interessan-

serva Ceserani che nella letteratura italiana contemporanea la modernità si è identificata soprattutto nell'espressionismo linguistico, dando luogo agli esiti di Gadda e, in parte, nell'espressionismo tematico, riflesso, per esempio, nelle opere di Svevo, Borgese, Pirandello, Bontempelli: «la cultura italiana e le sue istituzioni letterarie hanno reso difficile l'esplorazione dei temi postmoderni e delle nuove modalità rappresentative» (Cesarani 1997: 164). Il postmodernismo russo, invece, genera un'ampia dimensione culturale che contempla vari orientamenti estetici, i quali stabiliscono con la contemporaneità rapporti sia sincronici sia diacronici, dunque recuperando istanze culturali - quelle del Rinascimento, dell'Esistenzialismo, del Surrealismo, per esempio - precedentemente non elaborate dal discorso letterario russo. Lo studioso Mark Lipoveckij individua la presenza di due correnti postmoderniste: il concettualismo - tra i cui esponenti si possono individuare per esempio Dmitrij Prigov, Vladimir Sorovok, Lev Rubinštejn e Vsevolod Nekrasov - e il neobarocco, che si esprime nelle opere di Andrej Bitov, Saša Sokolov, Venedikt Erofeev. Negli anni Novanta i vari e complessi codici culturali del postmodernismo vengono introdotti nella cultura di massa e il successo commerciale dei romanzi di Viktor Pelevin e Vladimir Sorokin o di B.Akunin è prova della inesauribile fecondità del postmodernismo nella sua complessità (Lipoveckij 2001).

15Di Primo Levi verranno pubblicati dalla casa editrice Tekst di Mosca e tradotti entrambi dalle stesse traduttrici, E. Dmitrieva e I. Šubina La tregua (Peredyška, 2002 e 2011) e Il sistema periodico (Periodičeskaja sistema, 2008).

$16 \mathrm{Vi}$ è una corposa produzione poetica, saggistico-letteraria e linguistico-filologica, oltre che, naturalmente, narrativa. Per un puntuale resoconto bibliografico in argomento si veda: (Kazak 1996).

17 Tra altri titoli tradotti in russo figurano: Castelli di rabbbia; City; Emmaus; La storia di Don Giovanni; Mr Gwyn; Novecento; Omero, Iliade; Questa storia; Senza sangue; Tre volte all'alba. La popolarità dello scrittore presso i lettori russi è testimoniata dalla presenza di un sito in lingua russa dedicato al suo profilo e alla sua opera. 
te tendenza stilistica e tematica è Aldo Nove, con il romanzo Superwoobinda (trad. G. Kiselev, Moskva, Ad Marginem), annoverato nel sottogenere splatterpunk ${ }^{18}$, noto in Russia come fenomeno di importazione culturale, e letteraria in particolare, più che espressione autonomamente elaborata. Tale genere narrativizza la violenza della società, rea di generare psicopatici nascosti dalla maschera di rispettabili cittadini: «quello descritto dagli spltterpunk è il mondo visto dalla parte di Caino, è il male che osserva la realtà e la propria» (Mollo 2004: .96). Anche di Ammaniti viene pubblicata un'opera splatterpunk, la raccolta Fango (Grjaz'. Sbornik novell, trad. E. Žirnova, Sankt Peterburg, Symposium), nella quale si esprime il connubio eros e thanatos. Se, tuttavia, di Nove non viene pubblicata alcuna altra opera, neppure i suoi esiti appartenenti alla produzione successiva e più intimista che si colloca dopo l'anno 2000, di Ammaniti, sempre nel 2001, viene pubblicata la versione russa del romanzo Io non ho paura (Ja ne bojus', trad. V. Nikolaev, Moskva, Machaon), cui seguiranno, nel 2009, Come Dio comanda (Kak bog velit) e nel 2010 Che la festa cominci (Da budet prazdnik) entrambi tradotti da O. Uvarova e pubblicati dalla moscovita Inostranka.

Si può osservare che la produzione letteraria italiana degli anni Novanta approda all'editoria russa circa un decennio più tardi e che a partire dagli inizi degli anni Duemila la cultura russa accoglie le voci italiane più varie per espressione stilistica e tematica e per collocazione temporale. Accanto alla raffinata eleganza della prosa di Claudio Magris ${ }^{19}$, all'inclinazione surrealista di Luigi Malerba ${ }^{20}$ si esprimono, per esempio, la scabrosa comicità di Luciana Littizzetto ${ }^{21}$ e l'affabulazione storica di Valerio Massimo Manfredi, la cui presenza nell'editoria russa esordisce nel 2003 con Aleksandros (Aleksandr Makedonskij. Syn snovidenija, trad. M. Kononov, Moskva, ESKMO), per affermarsi negli anni successivi, con le edizioni dei suoi romanzi ${ }^{22}$.

Il pubblico russo apprezza le narrazioni di Margaret Mazzantini ${ }^{23}$, forse riconoscendovi quel tono antisentimentale per raccontare i sentimenti, quello stile preciso scultoreo, con qualche scabrosità in più, tuttavia, dei

18 Nove viene considerato un esponente della gioventù cannibale degli anni Novanta (dal titolo dell'antologia Gioventù cannibale pubbicata da Einaudi nel 1996). In argomento si veda: (Mondello 2004).

19 Del quale appaiono nel 2005 Un altro mare (Drugoe more, trad. V. Ljubin, Moskva, Symposium) e, nel 2012 Alla cieca (Vslepuju, trad. A. Dolgich, Moskva, Reka vremën).

$20 \mathrm{Nel} 2003$ viene pubblicato il romanzo Il serpente (Smeja, trad. F. Dvin, Moskva, Machaon) e nel 2008 Fantasmi romani (Rimskie prizraki, trad. F. Dvin, Moskva, Inostranka).

21Risale al 2006 il suo incontro con il pubblico russo, con la pubblicazione della raccolta di brevi racconti intitolata Sola come un gambo di sedano, nuovamente edito nel 2008 e nel 2011(Odna kak stebel' sel'derja, trad. E. Solonovič, Moskva, RIPOL).

22 Per l'elenco completo delle opere di Valerio Massimo Manfredi e degli altri autori ialiani pubblicati in Russia si rimanda al già citato : (Pavan 2014).

23 Appaiono nel 2005 Non ti muovere (Radi boga, ne dvigajsja, trad. Ju. Il'in, Sankt Peterburg, Azbuka klassika), riedito negli anni 2007, 2008, 2011 e 2013) e, nel 2011, Venuto al mondo (Roždennyj, trad. I. Bočenkova e N. Simonova, Sankt Peterburg, Azbuka), riedito nel 2012. 
romanzi di Ljudmila Ulickaja; mentre, consonante con l'orientamento neonaturalista russo, accompagnato dalla sempre più marcata indefinitezza, in termini di presenza e di funzioni, della voce narrante, con l'ibridazione tra fiction, non-fiction, faction è il romanzo Gomorra di Roberto Saviano (Gomorra, trad. Ja.Ar'kova, Moskva, Geleos, 2009), che costituisce «una sorta di limite, in senso matematico, della narrativa di inchiesta, che vede confermato il suo bisogno di verità extra-letteraria e quindi ridotto al minimo quello di perfezione compositiva» (Casadei 2013: 24).

La letteratura italiana contemporanea è oggetto di ricerca e di studio nei suoi aspetti stilisticamente più connotati e tematicamente più vari, motivo ispiratore di interessanti iniziative editoriali. È il caso della raccolta Ital' janskaja novella. XXI vek. Načalo ${ }^{24}$, a cura di E.Baglioni, autrice dell'introduzione, e di A. Černov (Moskva, Centr knigi Rudomino). L'antologia si presenta come «una finestra che affaccia sulla piccola prosa dell'Italia contemporanea» (Baglioni 2011:7). Importante è inoltre il ruolo svolto dalle riviste letterarie, in particolare da «Inostrannaja literatura», che alla letteratura italiana in Russia ha dedicato due corposi numeri monografici nel 2008, Ital' janskaja literatura $v$ poiskach formy (La letteratura italiana alla ricerca di una forma), a cura di A. Jampol'skaja, e nel 2011, Italija: vremena goda (Italia: le stagioni), a cura di E. Solonovič e Anna Jampol'skaja ${ }^{25}$. Infine è importante ricordare la fondamentale attività svolta dal complesso sistema editoriale russo, al quale si devono scelte, spesso coraggiose, investimenti - non economici soltanto, bensì anche culturali - passioni, orientate ad alimentare e a rinnovare il dialogo fra le lettere russe e le lettere italiane ${ }^{26}$.

Tra i numerosi editori citati nel presente contributo può essere interessante soffermarsi, seppure brevemente, su alcuni fra i più ricorrenti. La casa editrice Inostranka, per esempio, legata alla rivista «Inostrannaja literatura», si pone l'obiettivo di diffondere opere straniere di svariati generi, dalla letteratura di stampo intellettuale alla letteratura di intrattenimento nei suoi più popolari sottogeneri. Avvalendosi di abili traduttori, ha permesso al pubblico russo di conoscere autori come Michel Houellebecq, Jean-Christophe Grangé, Jonathan Franzen, Cecilia Ahem. Anche il nome Symposium ricorre frequentemente tra le case editrici interessate alla letteratura italiana. Fondata nel 1994, si orienta verso la pubblicazione di narrativa e, in particolare, di opere di carattere latamente umanistico, in prevalenza straniere. Tra gli autori pubblicati figurano Vladimir Nabokov, Yukio Mishima, Roman Gary, Peter Høeg. Due grandi grup-

24 L'antologia comprende racconti di Piergianni Curti, Flavia Ganzenua, Giorgio Falco, Giorgio Vasta, Ade Zeno, Alessandro De Roma, Elena Mearini, Sparajurij, Emanuele Tonon, Franco Arminio.

25 La puntuale descrizione dei contenuti dei due numeri monografici è oggetto di un articolo di C. Lasorsa Siedina (Lasorsa Siedina 2012).

26 Per una presentazione generale delle case editrici russe e delle relative iniziative volte a diffondere titoli di autori italiani si veda: ( Jampol'skaja 2016). 
pi editoriali nei cui cataloghi compaiono numerosi titoli italiani sono Azbuka ed Eksmo. La prima, fondata nel'95, vanta nel suo catalogo più di cento collane editoriali, alle quali si aggiungono pubblicazioni a sé stanti. Tra gli ambiti di interesse figurano la letteratura russa e straniera, classica e contemporanea, rappresentate da autori come Sergej Dovlatov, Iosif Brodskij, Milan Kundera, Patrick Süskind, Pascal Quignard. Eksmo si impone sul mercato editoriale russo a partire dal '91 grazie alla diffusione di titoli e di generi apprezzati dal grande pubblico come Aleksandra Marinina, Il'ja Derevjanko, autori, rispettivamente, di romanzi polizieschi e di thriller, e Dar'ja Doncova, nota per i suoi polizieschi dal carattere umoristico. Di grandi dimensioni è anche l'impresa Centrpoligraf, le cui edizioni sono probabilmente le più diffuse; i titoli in catalogo sono circa tremila e la tiratura annuale dei volumi editi supera i dodici milioni di copie. La sua strategia editoriale si orienta verso selezioni di opere appartenenti ai generi più apprezzati: polizieschi, storie criminali, fantasy, spy stories, biografie di attori, personaggi politici, ecc.

\section{BIBLIOGRAFIA}

Baglioni Elisa. "Vvedenie". Ital'janskaja novella XX vek. Načalo. Moskva: Centr Knigi Rudomino, 2011. pp. 5-11.

Caramitti Mario. Letteratura russa contemporanea. La scrittura come resistenza. Roma-Bari: Laterza, 2010. pp. 342.

Casadei Alberto. "Il grande romanzo italiano non esiste?" Somigli Luca. Negli archivi e per le strade. Il ritorno alla realtà nella narrativa di inizio millennio. Roma: Aracne, 2013. pp. 3-44.

Cesarani Remo. Raccontare il postmoderno. Torino: Bollati Boringhieri, 1997. pp. 242.

Dobrovolskaja Julija. Post scriptum. Memorie. O quasi. Venezia: Cafoscarina, 2006, pp.314

Jampolskaja Anna. "La letteratura italiana in Russia: osservazioni sulla politica editoriale". Palusci Oriana, Russo Katherine E. Translating East and West. Trento: Tangram Edizioni Scientifiche, 2016. pp.167-178.

Kozack Wolfgang. "La letteratura russa riunificata. Dieci anni di risanamento 1985-1994" Europa orientalis, 13, 1994, 2. pp. 189-208.

Kozak Wolfgang. Leksikon russkoj literatury XX veka, Moskva: RIK Kul'tura, 1996. pp. 491.

Ivanova Natal'ja B. “Uskol'zajuščaja sovremennost'. Russkaja literatura XXXXI vekov: ot "vnekomplektnoj k postsovetskoj, a teper' i vsemirnoj". Voprosy literatury, (2007), 3, http://magazines.russ.ru/voplit/2007/3.

Lasorsa Siedina Claudia. "La letteratura italiana in Russia". Studi Slavistici, IX (2012). pp.193-198.

Sovremennaja Enciklopedija 2000, http//dic.academic.ru/contents.nsf/enclp/ 
Lipoveckij Mark. "Russian Literary Postmodernism in the 1990s". The Slavonic and East European Review. 79, 1, (2001). pp. 31-50.

Lotman Jurij. Cercare la strada. Modelli della cultura. Venezia: Marsilio, 1994. pp.106.

Mineralov Jurij. Istorija russkoj literatury 90e gody XX veka, Moskva: Vlados, 2002. pp.471.

Pavan Stefania. L'Italia in cirillico. Verona: Cierre, 2013. pp. 852.

Mollo Marianna. "Cyberpunk e splatterpunk. Le frontiere estreme della fantascienza e dell'horror". Mondello Elisabetta. La narrativa italiana degli anni Novanta. Roma: Meltemi, 2004. pp. 87-102.

Palumbo Raffaello. “Narrazioni spurie: letteratura della realtà nell'Italia contemporanea". MLN, 1, January 2011, Italian issue. pp.200-221.

Đulija Bazelika

Italijanska proza u Rusiji od kraja 20. do početka 21. veka

Rezime

U noći 31. decembra 1991. okončano je jedno dugo poglavlje ruske istorije. Sovjetski Savez je zvanično ispustio poslednji dah. Ujedno se i početna lažna nada konzumerizma, omogućena novim kapitalizmom, odrazila u nagloj promeni u kulturnim navikama, što je zatim podstaklo poletnu atmosferu. Književnost je verovatno zauvek izgubila svoju tradicionalnu kulturnu predvodničku ulogu; profesionalni pisac, maître a penser, i izraz moći, iščezao je, dok je njegovo mesto preuzeo veoma uticajan i složen autoritet, tj. televizija, sposobna da izvrši sav svoj uticaj u isto vreme neizbežan i često neprimećen. U novom ruskom društvu, prožetom konzumerističkom logikom, knjiga je kao kulturni predmet i u široj svesti ljudi postala imovina predodređena da proizvede profitabilnost.

Pojavili su se novi izdavači: oni su predložili nove žanrove, koji su pre samo nekoliko godina bili zvanično nepoznati sovjetskim čitaocima, a koji su se približavali fantaziju, ljubavnim romanima, detektivskim pričama, erotskoj, treš i, od jedne krajnosti do druge, mističkoj književnosti - žanrove koji su počeli da se uobličavaju već u drugoj polovini osamdesetih. Među njima je bila antiutopija, sagrađena na osnovama nacionalne istorije i savremenosti i dugo vremena podstrekivač protivčinjeničnog čitanja prošlosti, kada nije političko-književni žanr.

U tako složenom kompleksu, ponekad ispresecanom neodređenim i neretko kontradiktornim trendovima, može se pokazati interesantnim ako bismo pokušali da izdvojimo prisustvo italijanske književnosti, naročito kada se radi o savremenoj proznoj produkciji.

Tako se u postsovjetskom kulturnom miljeu ranih devedesetih uočava prisustvo jasnog kontinuiteta književnog dijaloga između ruske i italijanske kulture. U nekoliko godina koje su usledile nakon „raspada“, tj. trogodišnjeg perioda 1992-1994 po raspadu Sovjetskog Saveza, objavljivana su dela autora dobro poznatih ruskim čitaocima kao što su Luiđi Pirandelo i Alberto Moravija. Veoma je zanimljivo objavljivanje Šašinih dela zajedno sa delima još jednog značajnog italijanskog klasika dvade- 
setog veka, Dina Bucatija. Italijanske detektivske priče koje naginju sentimentalnom postale su popularne i predstavljane su ruskim čitaocima do kraja druge decenije novog veka - tu su Frutero i Lučentini, Šerbanenko i Đorđo Faleti. Godine 2001 započinje nova i interesantna era u dijalogu između ove dve književnosti: rusko izdavaštvo pozdravlja dela tri prilično različita autora, naime Prima Levija, Itala Kalvina i Alda Nove; prethodnica pravog zaokreta u recepciji italijanske književnosti u ruskom miljeu jeste objavljivanje romana Ime ruže Umberta Eka 1989. Isti uspeh postigao je Alesandro Bariko, čija su dela godišnje reizdavana do 2018.

Italijanska književna produkcija iz devedesetih našla je svoje pristanište na ruskom izdavačkom tržištu otprilike jednu deceniju kasnije i od početka ovog veka ruska kultura je otvorenih ruku primila najrazličitije italijanske glasove. Ipak je interesantno analizirati, iako ukratko, profil i kulturnu misiju glavnih ruskih izdavačkih kuća zainteresovanih za širenje italijanske književnosti u Rusiji.

Ključne reči: italijanska književnost, prevod, rusko izdavaštvo, ruska književnost 